\title{
RADIOACTIVE BEAM DIAGNOSTICS STATUS AND DEVELOPMENT AT THE SPIRAL FACILITY
}

\author{
F. Chautard, J.L. Baelde, F. Bucaille, P. Duneau, C. Galard, J.P. Le Blay, \\ J.M. Loyant, L. Martina, M. Ulrich \\ GANIL (DSM/CEA,IN2P3/CNRS), Caen, France
}

B. Launé, Institut de Physique Nucléaire (CNRS/IN2P3), Orsay,France

\section{Abstract}

In 2001 the first radioactive beam will be accelerated in the CIME cyclotron of the SPIRAL project at GANIL. In order to be able to tune such low intensity beams in the cyclotron (down to few particles per second), a silicon and a scintillator detectors are mounted on probes. They measure the beam energy and the beam phase/RF in the cyclotron as a function of the radius. Such fragile diagnostics are meant to be used routinely from the control room by non-specialists in instrumentation and in presence of various beam intensities. Therefore, a program is developed to control and secure the acquisition procedure, the measurements and the isochronism correction. Additional detectors are installed at a fixed position in the ejection line before the experimental areas. Additionally, a diamond detector is foreseen to be installed in the machine to be tested in order to ease the CIME operation.

\section{LOW ENERGY BEAM LINE}

Because of the moderate resolution of the first mass selection out of the source $(\mathrm{R}=250)$ done by a dipole, it is necessary to have dedicated diagnostics [1] to detect the remaining ions after selection. This is done by a station connected to the low energy transport line by a switchyard. It will identify the ions by their radioactive properties and give the production rates of the source. This ion beam characterisation will be communicated to the cyclotron diagnostics to allow the tuning of the isochronism.

\section{CYCLOTRON}

\subsection{General Layout}

The tuning of the isochronism (beam phase/RF in function of the radius in the cyclotron $\phi(r))$ in the CIME cyclotron required exotic diagnostics because of the low intensity beams accelerated.

Actually, different kind of detectors are implemented in the cyclotron (Fig. 1):
- A scintillator plastic BC418 from Eurisys ( $\phi 15$ $\mathrm{mm}$ ) on a radial probe,

- A silicon detector from Eurisys, $300 \mu \mathrm{m}$ thick and $15 \times 15 \mathrm{~mm}^{2}$ on a second radial probe,

- $\mathrm{A} \mathrm{BaF} 2$ detector close to the vacuum chamber in a fixed position measuring the radiation emitted from the interception of the beam by a probe.

- A scintillator and a Si detector, $300 \mu \mathrm{m}$ thick and $\phi=15 \mathrm{~mm}$ at the exit of the cyclotron at a fixed position.

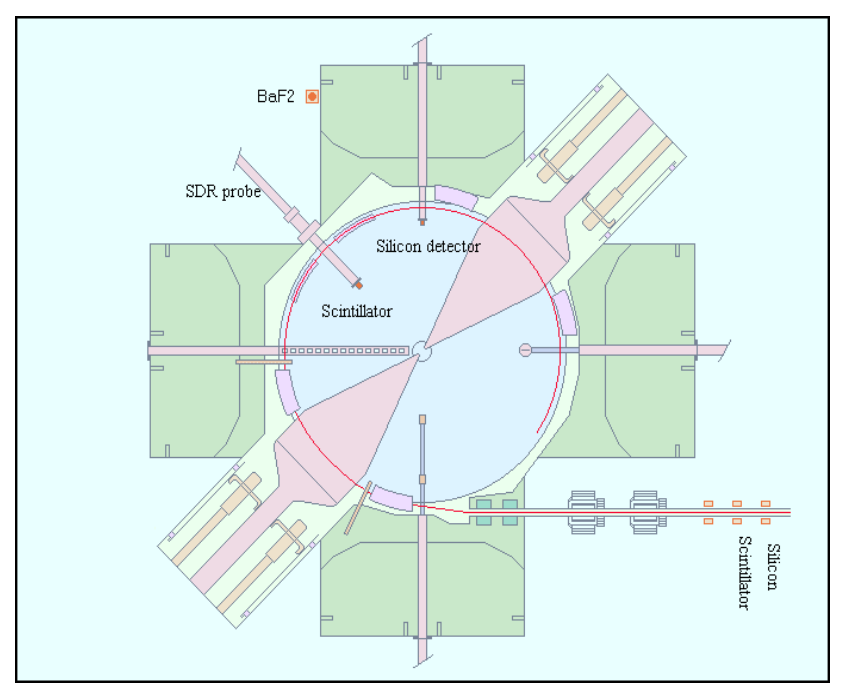

Figure 1: Detector positions in and around CIME

\subsection{Acquisition and control}

The analogic signals coming from the detectors (Fig. 2) are processed by a NIM electronic and converted to an ECL standard in order to be read by the VXI coders. A Fortran program does the control of the VXI processor from a standard VME station [2]. The nuclear physicists, to plot the data in one or two dimension spectra, use a Ganil program, called VISUGAN. This program has some useful features already implemented as contour, filtering, rms calculation etc.... But, because of the future utilisation of the acquisition system by non-expert, a 
graphical user interface (GUI) (Fig. 3) written in ADA [3] has been developed to combine:

- The equipment control (position and powering).

- The detector security control (beam flux limit).

- The VXI coders control.

- The calculation of the isochronism curve from a spectrum.

- The plot via VISUGAN of the results.

- The data transfer to a tuning program.

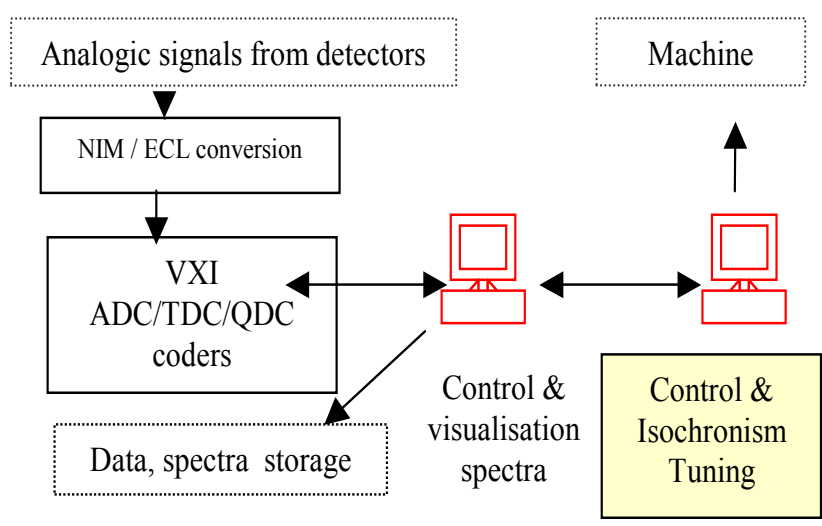

Figure 2: Schematic architecture of the exotic diagnostic acquisition system

Then, from the accelerator point of view, each command done previously from the VME station is done via the application.

This application handles also the detector safety by inserting the pepperpots in the injection line for each measurement to reach less than 1000 particles per second on the detectors. Additionally, if during the measurement the counting increases above this rate, a fast kicker deflects the beam before the cyclotron entrance and a beam-stop is inserted that can be removed only by a manual action from the control room. This situation may happen because of the presence of the pre-tuning ion necessary to tune the machine. Its intensity should be high enough to be able to use the classical detector. Its presence in the machine after the exotic beam tuning and an equipment drift might bring it onto the exotic detectors and destroy it

In the case of the detectors mounted on probes, three different acquisition modes are possible:

- Fixed position: when the rate or the detector efficiency are too low. The acquisition time is increased as well as the statistic.

- In flight (Fig. 4): there is an acquisition while the probe is moving. It is used when whether the counting is high enough or to have a first quick look at the spectrum before acquiring for statistic.
- $\quad$ Step-by-step (Fig. 5): The acquisition time for each step is entered, as well as the distance in millimetre between two measurements.

Another window is dedicated to the control of the VXI crate for initialisation, acquisition launch or acquisition stop.

An important feature is the possibility to choose an detector mode. When a mode is selected (scintillator probe, silicon probe, ejection scintillator, etc...) only events from the selected detectors are validated. Which eliminates noise or parasitic behaviour from the other detectors.

Finally and it is the main purpose of the program, this program should filter and extract the data of interest from a spectrum and fit a curve representing the isochronism law to be corrected. The equation of the curve will be sent to another existing GUI in order to calculate the correction current required in the eleven-isochronism coils and the main coil.

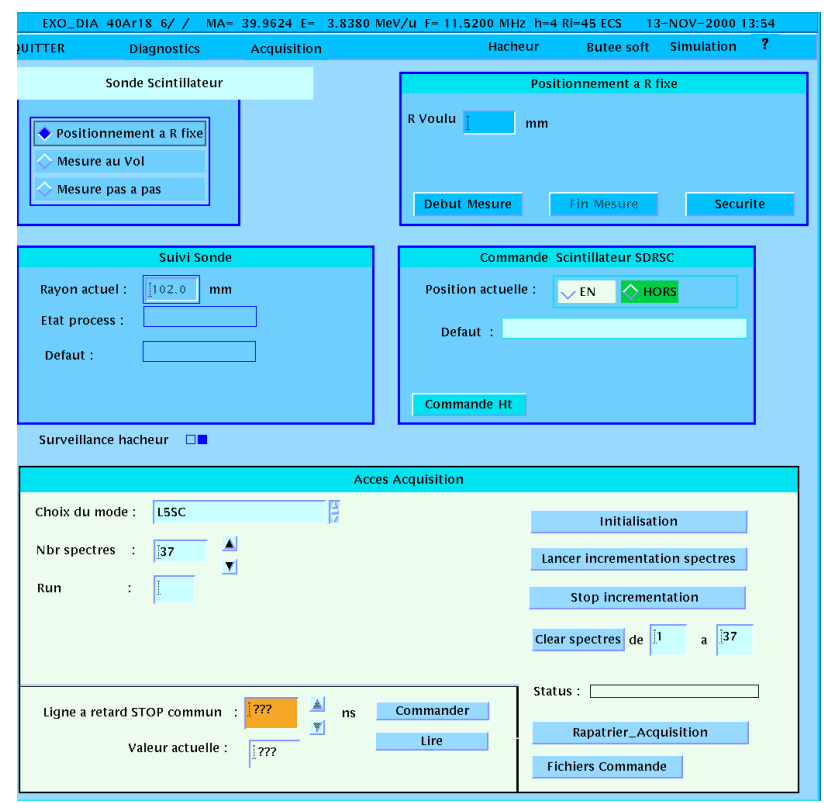

Figure 3: Display of graphical user interface EXO_DIA.

\section{MEASUREMENTS}

In the following, we show typical measurement examples done with the scintillator probe for $\mathrm{a}_{16} \mathrm{O}^{3+}$ beam at 12 $\mathrm{MHz}$ RF frequency on harmonic 3. The ejection energy was $7.4 \mathrm{MeV} / \mathrm{A}$. Figure 4 shows an in-flight acquisition. Due to the limited energy sensitivity of the plastic (1 or 2 $\mathrm{MeV} / \mathrm{A}$ in function of the ion mass), the beam cannot be seen before a radius of $800 \mathrm{~mm}$. Additionally, electric 
noise from the deflector is superimposed to the beam signal at ejection. Detector shielding should overcome this problem. As the isochronism curve is a smooth curve, only discrete points can be measured with longer acquisition time to increase the statistic. This is the measurement shown in Figure 5.

\section{CONCLUSIONS}

Presently, the exotic diagnostics are used routinely and formation is given to spread their use to the whole Ganil operation group. At the end of last year a preliminary experiment with nuclear physicists was done to study beam acceleration in CIME with several ion species from

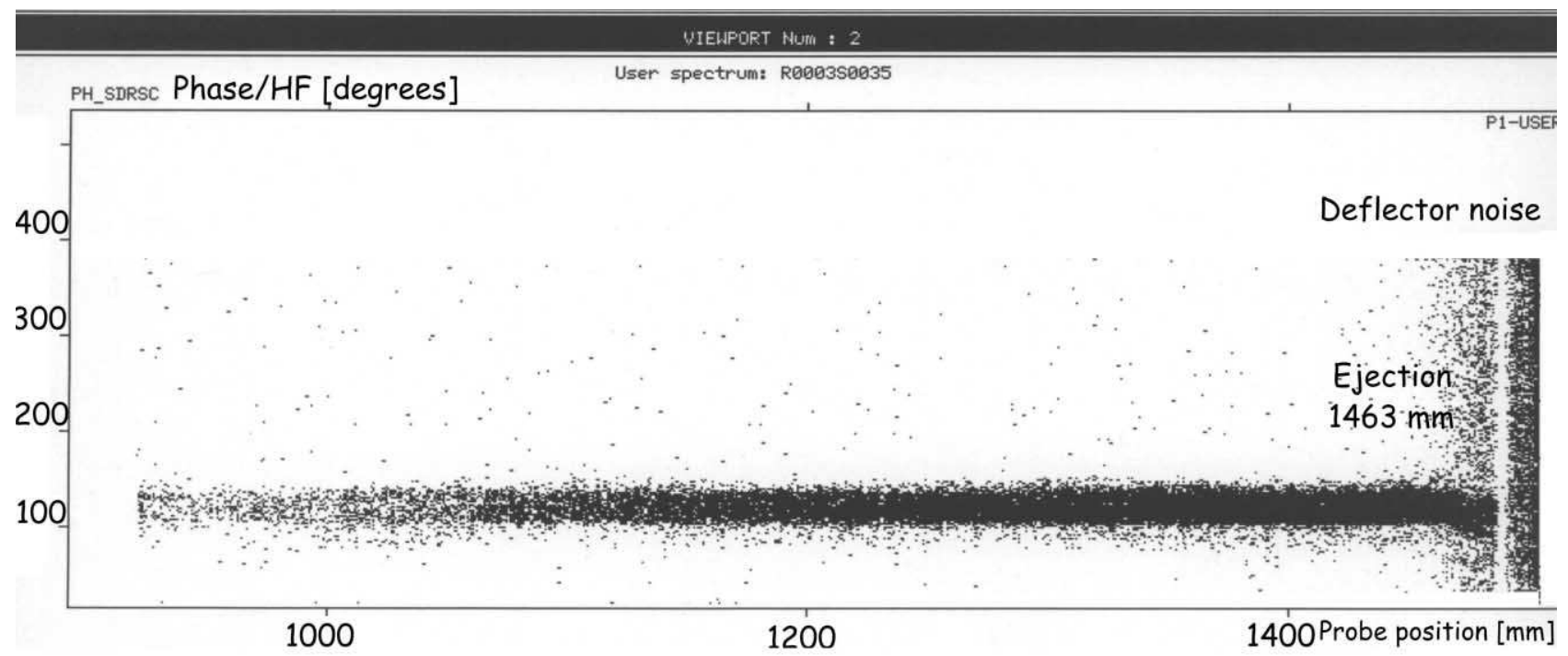

Figure 4: In-flight acquisition with a plastic detector.

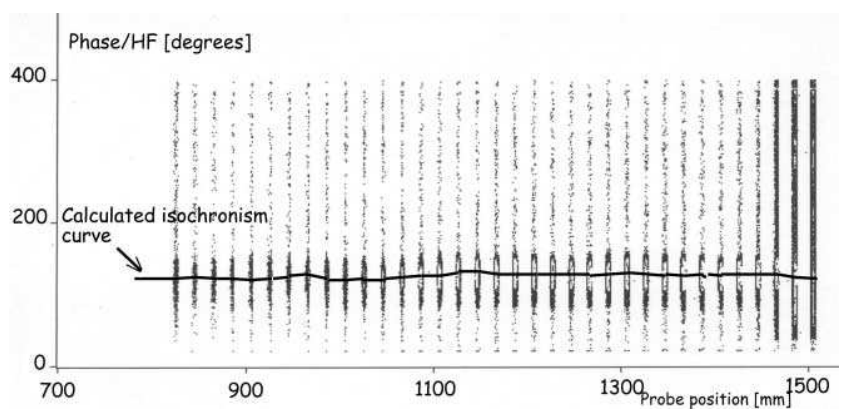

Figure 5: Plastic detector measurement with a step-bystep acquisition. An isochronism curve is superimposed.

\section{DEVELOPMENTS}

A large effort from the Accelerator Division is done to develop new detectors adapted to the operation of CIME. The diamond detector [4] with its good resistance to high particle flux and fast rise-time response makes a good candidate to be used by non-specialists from a control room. A collaboration is under way with the LIST from CEA/Saclay laboratory, France, to test the detector properties under different ion beams and see if it is well suited to our purpose.
Carbon to Xenon (Fig. 6). This fruitful collaboration allowed us to improve our technique. Then, new collaborations are foreseen and essential with

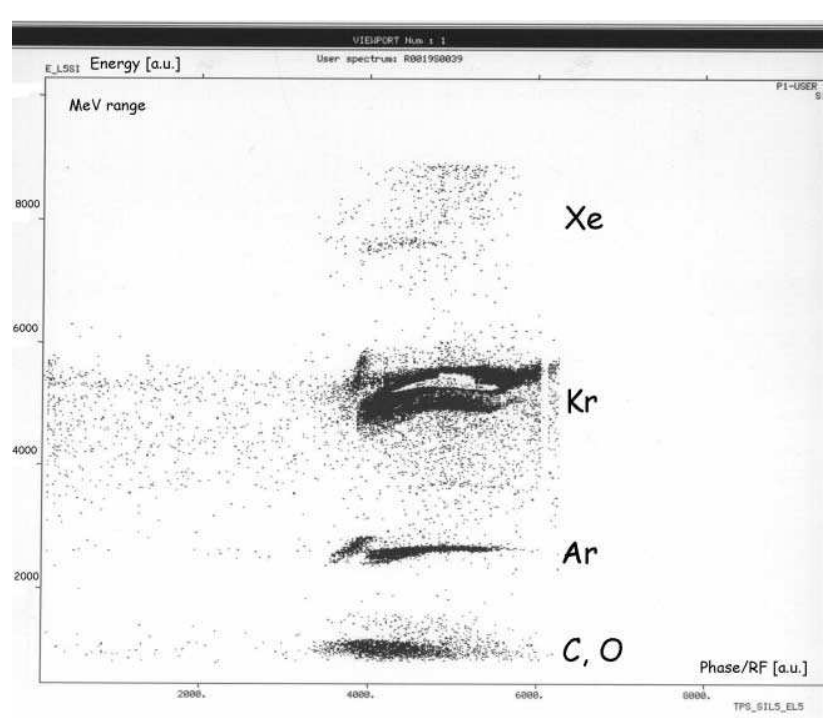

Figure 6: Energy measurement with a $300 \mu \mathrm{m}$ silicon detector at a fixed position in the cyclotron during frequency variation with several ions accelerated.

instrumentation specialists to enhance the development of such exotic equipments dedicated to accelerators. 


\section{REFERENCES}

[1] B. Launé, "Diagnostics for radioactive beams", this conference.

[2] B. Piquet et al., "The standard GANIL Data Acquisition System", Eigth conference on real-time computer application in nuclear, particle and plasma physics, Vancouver, 1993.

[3] Groupe informatique machine, "Exo_sondes", GANIL/DA-99.418A/CG.

[4] P. Bergonzo et al., "CVD Diamond for radiation detection", to be published in Diamond and Related Materials, April 2001. 